\title{
Detection of differences between visual textures with varying number of dots
}

\author{
COLIN V. NEWMAN* \\ University of Birmingham, Birmingham, England
}

\begin{abstract}
Sixty-four Ss were tested on a judgmental task involving discrimination between textures with variable numbers of elements. Results showed that the threshold for detecting percentage differences in numbers of dots in adjacent patterns decreased monotonically with the total number of dots in the standard. Implications of this finding for the perceptual processing of textures are discussed.
\end{abstract}

Interest in the role of texture as a variable in visual space perception has stemmed from the theory of Gibson (1950). More recently discussion has focused on the visual processes involved in perceiving texture itself (Pickett, 1967; Rosenfeld, 1967). Pickett (1968) has reviewed studies concerning the detection of differences between textures in which several parameters of the textures, including number of elements, size and density of elements, area of display, and exposure time, have been varied. The majority of these experiments, such as that by Green, Wolf, and White (1959), have used computer-generated textures composed of several hundred or thousand dots. With such displays, Pickett (1968) concluded that there are psychophysically detectable shifts in perceptual processing as pattern complexity is increased. The present experiment extends the analysis of texture discrimination to emergent or rudimentary textures by using dot displays of many fewer elements than those of most previous experiments.

One experiment reported by Underwood (1966) tested discrimination judgments with simple dot displays having between 16 and 128 dots in the standard. As number of dots in the standard increased, the number of dots in the comparison required to produce a discriminable difference increased. In proportional terms, however, the threshold for detecting a percentage difference in dot number remained constant in accordance with the expectations of Weber's law, except for the 16-dot standard. Underwood suggested that this discrepant result with the 16-dot standard occurred because Ss had sufficient time to actually count the dots or estimate the number with high accuracy. Underwood's experiment thus provided no evidence for any psychophysically detectable shift in texture discrimination as dot number was increased. The present experiment reexamined the issue of detecting differences between textures. Dot displays composed of similar, small numbers of dots were used, but the standard and comparison displays were presented simultaneously and not successively as in the experiment by Underwood.

*Requests for reprints should be sent to Colin V. Newman at the Department of Psychology, University of Birmingham, P.O. Box 363, Birmingham B15 2TT, England.

\section{METHOD}

Thirty-five pairs of stimulus patterns were prepared on white cards, $15 \times 10 \mathrm{~cm}$. On each card, two adjacent rectangles were drawn in dark ink, each $6 \times 5 \mathrm{~cm}$ and sharing a common $6-\mathrm{cm}$ side. A standard number of $20,40,60,80$, or 100 black dots was drawn in one rectangle, and a comparison series of identical black dots was drawn in the adjacent rectangle. Each comparison series contained one rectangle with the same number of dots as the standard and six further rectangles containing progressively fewer dots than the standard, decreasing in steps of 5\% compared with the standard. This yielded 35 pairs of adjacent textures containing dots as follows: $(20,20),(20,19),(20,18)$, $(20,17),(20,16),(20,15),(20,14) ;(40,40),(40,38),(40$, $36), \ldots,(40,28) ;(60,60),(60,57), \ldots,(60,42) ;(80,80), \ldots$, $(80,56) ;(100,100), \ldots,(100,70)$. The required number of 10-cm-diam dots was scattered at random within each rectangle except for one constraint, namely, that no dots should actually touch each other or a boundary line.

To present the patterns to Ss, the data transmission system of the Department of Psychology, University of Birmingham, was used. The Ss sat separately in 16 cubicles, $1 \mathrm{~m}$ from television screens on which the pairs of adjacent patterns were displayed, at eye level, for $2 \mathrm{sec}$ each. Exposure duration was controlled by tachistoscopic presentation of stimulus cards to a television camera in a control room. The $\mathrm{E}$ warned Ss of ensuing presentations and gave instructions over an intercom system with loudspeakers in each cubicle. The television pictures of stimulus cards were adjusted to have the same physical dimensions as the actual cards.

Sixty-four Ss of both sexes with normal or corrected-to-normal vision were tested in four identical experimental sessions. The Ss were undergraduate psychology students, naive as to the predictions of the experiment. The Ss were instructed on each trial to judge whether the rectangle presented on the left or the right contained more dots or whether both rectangles contained an equal number of dots. Judgments were recorded by Ss on score sheets. The Ss made a total of 140 discrimination judgments. The entire series of 35 cards was presented a first time in random order with the left or right position of the standards on each card also being randomized. The series of 35 cards was then presented in further random orders for three more series of discrimination judgments. For the second and third series, all standards that had initially been presented on the left were presented on the right, and vice versa, for standards initially presented on the right. The last series was identical to the first so that every card was viewed in all four times, twice with the standard to the left and twice to the right. In accordance with the principles of the psychophysical method of constant stimulus differences, for each value of the standard the threshold was calculated for detecting a difference in number of dots in adjacent rectangles. 
Table 1

Mean Percentage Detection Threshold for Different Standard Numbers of Dots

\begin{tabular}{lcrrrr}
\hline Number of Dots in Standard & 20 & 40 & 60 & 80 & 100 \\
\hline Mean Percentage Detection Threshold & 13.36 & 12.80 & 11.84 & 11.52 & 10.11 \\
Standard Deviation & \pm 5.61 & \pm 4.63 & \pm 4.94 & \pm 4.15 & \pm 4.26 \\
\hline
\end{tabular}

For each standard number of dots, each S's threshold was expressed as the percentage decrease in the number of dots in the comparison for a difference in dot numbers to be detected.

\section{RESULTS}

Table 1 shows for each standard the threshold mean percentage difference in number of dots.

When compared by one-way analysis of variance, these means were significantly different, $F(4,252)=$ $6.53, \mathrm{p}<.01$. Trend analysis of the means yielded a large and significant linear component, $\mathrm{F}(1,63)=28.76$, $\mathrm{p}<.01$, with very small and nonsignificant quadratic, cubic, and quartic components.

\section{DISCUSSION}

The results were unequivocal: the threshold for detecting percentage differences in numbers of dots in adjacent patterns decreased monotonically with the total number of dots in the standard. This result was at variance with that reported by Underwood (1966); however, the experiments used different sizes of dots and patterns of different area. Temporal differences in the method of presenting the standard and comparison have already been described; furthermore, in both experiments, manipulation of the number of dots present in the variable stimulus simultaneously altered other aspects of the stimulus such as average separation between dots. The data must be interpreted with caution until full parametric experiments manipulating all relevant variables have determined the conditions under which the effect shown in this experiment is found to hold. Despite this caution, the results imply that under certain conditions, threshold for detecting differences in number of dots in adjacent textures decrease as number of dots increase to give an emergent texture. Although no sudden shifts in thresholds were found, the results supplement those of Pickett (1964), who with a more ecologically representative range of textures composed of more numerous elements did find evidence for psychophysically detectable shifts in perceptual processing as pattern complexity was increased. If confirmed in other experiments, such evidence for variations or shifts in perceptual processing with increases in pattern complexity would have implications for the role of surface texture in space perception. When gradients of texture density provide information for spatial judgments, the visual system must process the relative densities with which similar texture elements occur in different portions of the visual field. Gibson $(1950,1966)$ has not indicated when a texture is above threshold or when it contains sufficient elements to provide information for perceiving the layout of surfaces. The present results suggesting variations in perceptual processing with complexity of texture would imply different information extraction possibilities as textures become more complex. Further experiments are needed to investigate the relationship between the precision with which differences between textures with different measurable parameters such as number and density of elements can be detected and the accuracy with which various facets of surface layout such as surface slant or surface length can be judged.

\section{REFERENCES}

Gibson, J. J. The perception of the visual world. Boston: Houghton Mifflin, 1950.

Gibson, J. J. The senses considered as perceptual systems. Boston: Houghton Mifflin, 1966.

Green, B. F., Wolf, A. K., \& White, B. W. The detection of statistically defined patterns in a matrix of dots. American Journal of Psychology, 1959, 72, 503-520.

Pickett, R. M. The perception of visual texture. Journal of Experimental Psychology, 1964, 68, 13-20.

Pickett, $R$. M. The perception of random texture. In $W$. Wathen-Dunn (Ed.), Models for the perception of speech and visual form. Cambridge, Mass: M.I.T. Press, 1967.

Pickett, R. M. Perceiving visual texture: A literature survey. Technical Report 68-12, 1968, Aerospace Medical Research Laboratories, Wright-Patterson Air Force Base, Ohio.

Rosenfeld, A. On models for the perception of visual texture. In W. Wathen-Dunn (Ed.), Models for the perception of speech and visual form. Cambridge, Mass: M.I.T. Press, 1967.

Underwood, B. J. Experimental psychology. New York: Appleton-Century-Crofts, 1966. Pp. 171-175.

(Received for publication June 5, 1974.) 